\title{
Análises da precipitação pluvial no Estado da Paraíba com base na teoria da entropia
}

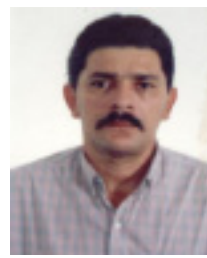

Vicente de P. R. da Silva ${ }^{1}$, Enilson P. Cavalcanti ${ }^{2}$, Marília G. do Nascimento ${ }^{3}$ \& João Hugo B. da C. Campos ${ }^{3}$

\author{
DCA/CCT/UFCG. Fone: (83) 310-1202. E-mail vicente@dca.ufpb.br (Foto) \\ 2 DCA/CCT/UFCG. Av. Aprígio Veloso 882, CEP 58109-970, Campina Grande, PB. E-mail: enilson@dca.ufpb.br \\ ${ }^{3}$ Bolsista do Programa Institucional de Bolsas de Iniciação Científica da UFCG
}

Protocolo 182 - 28/11/2002 - Aprovado em 31/7/2003

\begin{abstract}
Resumo: Os dados diários de precipitação pluvial de 58 postos pluviométricos do Estado da Paraíba e de temperatura média diária de Campina Grande, PB, foram utilizados para avaliar a variabilidade espacial e temporal da precipitação pluvial, com base na teoria da entropia. Analisouse, também, a variabilidade temporal da temperatura do ar em Campina Grande, com base nessa mesma teoria. Os resultados evidenciaram que a entropia é alta em locais com intensa precipitação, e baixa quando ocorrem, nesses locais, baixos índices pluviométricos; conseqüentemente, nos períodos chuvosos a entropia é alta e, nos períodos de estiagem, é mínima. Este trabalho evidencia, ainda, que qualquer série temporal de entropia decresce exponencialmente com o aumento do seu desvio-padrão.
\end{abstract}

Palavras-chave: entropia de Shannon, precipitação diária, temperatura do ar

\section{Use of entropy theory in analysis of rainfall and air temperature}

\begin{abstract}
The objective of this study was the evaluation of the rainfall variability in Paraíba state. Daily rainfall observed from a network of 58 rain gauges in Paraíba State and mean daily temperature observed at Campina Grande weather station were analyzed using entropy theory. The results showed that rainfall entropy is high in localities and periods with high rainfall values and is low in localities and periods with low rainfall values. Maximum values of rainfall entropy occurred in the rainy period and minimum values occurred in the dry period. However, the entropy of the temperature time serie was constant, with maximum value of 8.53 bits. This study shows that for any time series the entropy decreases exponentially with increase of standard deviation.
\end{abstract}

Key words: Shannon's entropy, daily rainfall, air temperature

\section{INTRODUÇÃO}

A precipitação pluvial média anual no semi-árido do Estado da Paraíba é inferior a $800 \mathrm{~mm}$ e, na parte litorânea, os totais anuais podem superar os $1500 \mathrm{~mm}$. A variação espacial da precipitação pluvial no Estado é provocada por diferentes sistemas atmosféricos que atuam na costa Leste do Nordeste do Brasil. Roucou et al. (1996) observaram que a precipitação pluvial no Nordeste do Brasil também é associada aos movimentos verticais ascendentes de ar e à migração da Zona de Convergência Intertropical (ZCIT). As distribuições anual e intra-anual das chuvas nas microrregiões do Estado da Paraíba são muito semelhantes àquelas das microrregiões circunvizinhas; apesar de sua pequena área $\left(56.584,6 \mathrm{~km}^{2}\right)$, a Paraíba apresenta estações chuvosas bem definidas, ou seja, no Sertão de janeiro a março, no Cariri e Agreste de março a maio e na Mata Paraibana de abril a junho (Silva et al., 1996).
O termo entropia, como conceito científico, é originário da Física e foi inicialmente utilizado em termodinâmica por Clausius, em 1850. Sua interpretação probabilística, dentro da mecânica estatística, é atribuída a Boltzmann em 1877, sendo o relacionamento com probabilidade registrado vários anos depois, em 1906, por Planck. Mais recentemente, Shannon \& Weaver (1949) utilizaram o conceito de entropia em análise econômica e na solução de problemas relacionados à teoria de codificação e transmissão de dados. Esta contribuição à teoria moderna da informação tem sido aplicada, atualmente, em diversas áreas do conhecimento, como hidrologia (Singh, 1997), matemática (Dragomir et al., 2000), economia (Kaberger \& Mansson, 2001), ecologia (Ricotta, 2001), climatologia (Kawachi et al., 2001) e medicina (Montaño et al., 2001).

Para entender o aspecto informacional da entropia, devese considerar um conjunto de $\mathrm{n}$ elementos, sendo a incerteza da ocorrência de um deles considerada como a situação na 
qual não se conhece qual deles ocorrerá (Singh, 1997). Com base no conhecimento de um único evento, a incerteza poderá ser maior ou menor; por exemplo, o número total de eventos $n$ é uma parte da informação e o número desses eventos com probabilidade diferente de zero é outra parte da informação. Esta distribuição de probabilidade, se conhecida, oferece um número de informações que pode reduzir as incertezas associadas ao sistema. Desta forma, a incerteza pode ser quantificada pela entropia, levando-se em conta todos os tipos de informações disponíveis, e representada pela distribuição de probabilidade da variável considerada. Interpretando um conjunto composto por $n$ eventos e se considerando $p_{i}$ como a probabilidade de encontrar o sistema no enésimo microestado, a entropia de Shannon é igual à entropia da mecânica estatística.

Chapman (1986) aplicou o conceito de entropia condicional para comparar a eficiência de modelos hidrológicos. Rajagopal et al. (1987) apresentaram novas perspectivas para aplicação potencial da entropia em pesquisas relacionadas com recursos hídricos. Sonuga (1976) descreveu a aplicação do princípio da entropia no processo de modelagem de chuva-vazão, e observou que essa técnica pode ser utilizada quando a disponibilidade de dados é mínima. Kawachi et al. (2001) utilizaram o conceito de entropia para avaliar o grau de variabilidade da precipitação pluvial no Japão e obtiveram mapas de disponibilidade hídrica.

As incertezas associadas às séries temporais podem ser estimadas quantitativamente, utilizando-se a teoria da entropia. Esta técnica tem sido aplicada para determinar a incerteza da intensidade e a quantidade da precipitação pluvial (Kawachi et al., 2001). Em estudos relacionados com essa variável climatológica, as probabilidades são definidas como a freqüência de ocorrência da quantidade discreta da precipitação distribuída sobre certo período de tempo. De acordo com esta definição, a entropia nula ocorre quando a intensidade da precipitação é uniforme no tempo.

A variabilidade espacial e temporal da precipitação pluvial, face à sua incerteza e irregularidade ao longo do tempo, constitui-se num problema crucial em estudos climatológicos. Esta variabilidade é ainda maior em regiões tropicais, particularmente no Nordeste do Brasil, onde atuam vários sistemas atmosféricos, como a zona de convergência intertropical, os sistemas frontais, as brisas de leste e os vórtices ciclônicos. Por outro lado, a temperatura média do ar apresenta baixa variabilidade, sendo, portanto, facilmente modelada em função das coordenadas geográficas, com alto nível de confiabilidade (Cavalcanti \& Silva, 1994). O desenvolvimento da teoria da entropia por Shannon \& Weaver, em 1949, e o princípio da máxima entropia proposto por Janes, em 1950, têm sido amplamente utilizados em ciências ambientais em diversos países do mundo; entretanto, no Brasil, essa metodologia é ainda incipiente.

Neste sentido, o presente trabalho objetivou analisar o grau de variabilidade espacial e temporal da precipitação pluvial do Estado da Paraíba; quantificar a variação temporal da precipitação pluvial e da temperatura do ar em Campina Grande, PB, e obter a relação entre entropia e o desvio-padrão de uma série temporal.

\section{MATERIAL E MÉTODOS}

Neste trabalho foram utilizados dados diários de 58 postos pluviométricos localizados no Estado da Paraíba, com séries de 10 anos de dados. O período de estudo de cada série variou em função do critério de se utilizar apenas aquelas séries com 10 anos de dados contínuos e absolutamente sem falhas. Foram também empregados os dados diários de precipitação pluvial, do período de 1939 a 1972, e de temperatura média do ar, do período de 1963 a 1972, da estação climatológica de Campina Grande, PB (latitude: $7^{0} 13^{\prime}$; longitude: $35^{\circ} 54^{\prime}$; altitude $508 \mathrm{~m}$ ). Os dados mais recentes de temperatura do ar e de precipitação pluvial não foram utilizados na presente análise porque não atenderam ao critério estabelecido acima. A Tabela 1 exibe a relação dos postos pluviométricos utilizados neste estudo, com suas respectivas coordenadas geográficas.

Tabela 1. Características das estações pluviométricas do Estado da Paraíba utilizadas no estudo

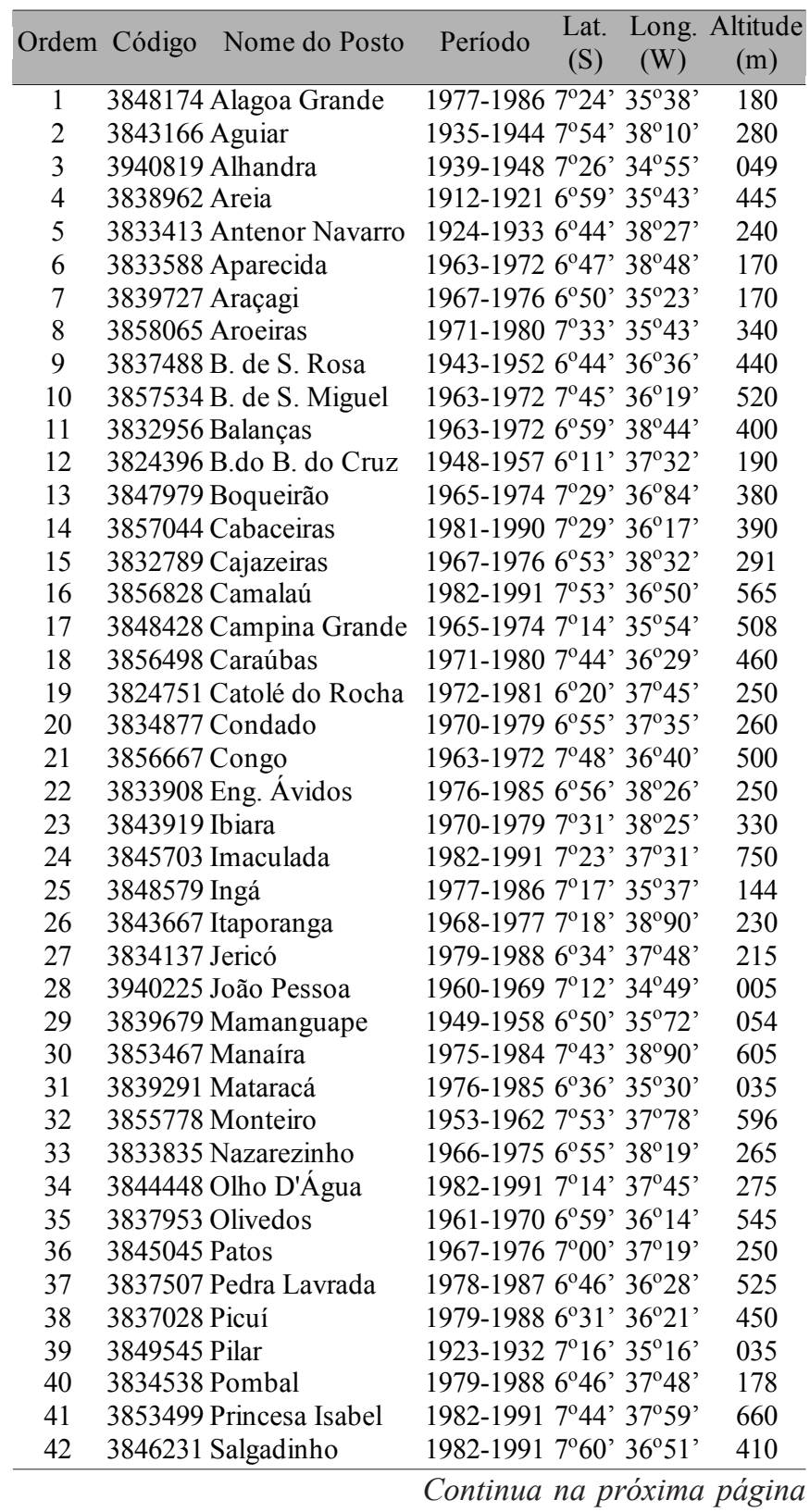


Continuação da Tabela 1

\begin{tabular}{|c|c|c|c|c|c|}
\hline Ordem & Nome do Posto & Período & $\begin{array}{l}\text { Lat. } \\
\text { (S) }\end{array}$ & $\begin{array}{l}\text { Long. } \\
\text { (W) }\end{array}$ & $\begin{array}{c}\text { Altitude } \\
(\mathrm{m})\end{array}$ \\
\hline 43 & 3846231 S. de S. Félix & 1979-1988 & $7^{0} 22{ }^{\prime}$ & $35^{\circ} 26^{\prime}$ & 400 \\
\hline 44 & 3836715 Santa Luzia & 1970-1979 & $6^{\circ} 52^{\prime}$ & $36^{\circ} 55^{\prime}$ & 290 \\
\hline 45 & 3846894 São J. do Cariri & 1959-1968 & $7^{\circ} 23^{\prime}$ & $36^{\circ} 32^{\prime}$ & 445 \\
\hline 46 & 3866128 São J. do Tigre & $1981-1990$ & $8^{\circ} 48^{\prime}$ & $36^{\circ} 51^{\prime}$ & 616 \\
\hline 47 & 3833869 S. J. de L. Tapad & $1981-1990$ & $6^{\circ} 56$ & $38^{\circ} 96^{\prime}$ & 260 \\
\hline 48 & 3835734 S. J. de Espinhara & 1979-1988 & $6^{\circ} 51^{\prime}$ & $37^{\circ} 20^{\prime}$ & 185 \\
\hline 49 & 3843202 S. J. de Piranhas & 1982-1991 & $7^{\circ} 72^{\prime}$ & $38^{\circ} 30^{\prime}$ & 300 \\
\hline 50 & 3835882 São Mamede & 1980-198 & $6^{\circ} 56^{\prime}$ & $37^{\circ} 60^{\prime}$ & 270 \\
\hline 51 & 3858467 S. S. Umbuzeiro & 1972-1981 & $8^{\circ} 90^{\prime}$ & $37^{\circ} 60^{\prime}$ & 600 \\
\hline 52 & 3837717 Seridó & 1976-1985 & $6^{\circ} 56^{\prime}$ & $36^{\circ} 23^{\prime}$ & 420 \\
\hline 53 & 3847128 Soledade & 1980-1989 & $7^{\circ} 36^{\prime}$ & $36^{\circ} 22^{\prime}$ & 560 \\
\hline 54 & 3856314 Sumé & $1965-1974$ & $7^{\circ} 40^{\prime}$ & $36^{\circ} 54^{\prime}$ & 510 \\
\hline 55 & 3846434 Taperoá & 1940-1949 & $7^{\circ} 13^{\prime}$ & $36^{\circ} 50^{\prime}$ & 500 \\
\hline 56 & 3845448 Teixeira & $1974-1983$ & $7^{\circ} 13^{\prime}$ & $37^{\circ} 15^{\prime}$ & 770 \\
\hline 57 & 3833018 Uirauna & $1979-1988$ & $6^{\circ} 31^{\prime}$ & $38^{\circ} 25^{\prime}$ & 300 \\
\hline 58 & 3858467 Umbuzeiro & $1970-1979$ & $7^{\circ} 42^{\prime}$ & $35^{\circ} 40^{\prime}$ & 553 \\
\hline
\end{tabular}

A entropia de uma variável, considerada como a estimativa da incerteza da ocorrência de determinado evento num processo aleatório discreto, pode ser obtida através da seguinte equação (Shannon \& Weaver, 1949):

$$
\mathrm{H}=-\mathrm{k} \sum \mathrm{p}_{\mathrm{i}} \log \mathrm{p}_{\mathrm{i}}
$$

em que:

$\mathrm{H}$ - entropia da variável aleatória discreta, bits

$\mathrm{p}_{\mathrm{i}}$ - probabilidade da enésima variável aleatória discreta, adimensional

$\mathrm{k}$ - constante positiva, cujo valor depende das unidades utilizadas

A unidade de entropia pode ser bit para a base logarítmica 2 , napiers ou nats para a base neperiana e hartley para a base 10. Neste trabalho utilizou-se a unidade bit para entropia, que significa dígito binário, ou seja, a menor unidade na notação numérica binária, que pode assumir o valor 0 ou 1 . Neste caso, assumindo-se a constante $\mathrm{k}$ com valor igual a $1 \mathrm{e}$ a base do logaritmo 2, a Eq. 1 pode ser simplificada para:

$$
\mathrm{H}=-\sum_{\mathrm{i}=1}^{\mathrm{n}} \mathrm{p}_{\mathrm{i}} \log _{2} \mathrm{p}_{\mathrm{i}}
$$

em que:

n - número possível de eventos da variável aleatória discreta, adimensional

Se todos os pi são iguais, isto é, se $p_{i}=1 / n$, então, $H=\log _{2} n$. Assim, $\mathrm{H}$ é uma função monotonicamente crescente em $\mathrm{n}$. A entropia é máxima quando todos os $\mathrm{pi}_{\mathrm{s}}$ são iguais; ao contrário, é mínima, e igual a zero, quando todos os pi s, exceto um, é zero, significando que todo resultado da variável aleatória é sempre o mesmo e, portanto, um dos pi torna-se unitário. Dessa forma, o valor da entropia varia dentro do intervalo de zero a $\log _{2} n$, de acordo com a forma da distribuição de probabilidade dos pi $\mathrm{s}_{\mathrm{s}} \mathrm{O}$ valor da entropia decresce com o aumento do número de contraste e aumenta com o decréscimo desse número. Visto desta maneira, a entropia pode ser considerada uma estimativa funcional da incerteza associada à distribuição de probabilidade.

Para cada série histórica de precipitação de um ano, será admitido que $r_{i}$ seja a precipitação pluvial diária correspondente ao enésimo dia do ano; por exemplo, valores diários da precipitação pluvial de 1 de janeiro a 31 de dezembro, para o mesmo ano, podem ser expressos por $\mathrm{r}_{1}$ e $\mathrm{r}_{365}$, respectivamente. Logo, a precipitação total durante $o$ ano $(\mathrm{R})$ pode ser expressa pelo somatório dos valores diários, variando de $\mathrm{i}=1$ até $\mathrm{i}=365$, por:

$$
\mathrm{R}=\sum_{\mathrm{i}=1}^{365} \mathrm{r}_{\mathrm{i}}
$$

Os valores de $r_{i}$ podem ser zero para alguns dias e diferentes de zero para outros dias. As séries de precipitação formadas por $\mathrm{r}_{1}, \mathrm{r}_{2}, \ldots, \mathrm{r}_{\mathrm{n}}$ podem, assim, ser fixadas como a freqüência de ocorrência acumulada de chuvas para $1,2, \ldots$, enésimo dia do ano, respectivamente. Assim, a freqüência relativa da precipitação $\left(\mathrm{p}_{\mathrm{i}}\right)$ será obtida dividindo-se $\mathrm{r}_{\mathrm{i}}$ pelo tamanho total da amostra (R), ou seja:

$$
p_{i}=\frac{r_{i}}{R}
$$

A freqüência relativa $\left(\mathrm{p}_{\mathrm{i}}\right)$ será fixada como uma probabilidade de ocorrência do total da precipitação no enésimo dia e, portanto, sua distribuição representa a característica probabilística da partição temporal da precipitação ao longo do ano, isto é, a ocorrência da incerteza da precipitação. Substituindo-se a Eq. 4 na Eq. 2 obtém-se:

$$
\mathrm{H}=-\sum_{\mathrm{i}=1}^{\mathrm{n}} \frac{\mathrm{r}_{\mathrm{i}}}{\mathrm{R}} \log _{2}\left(\frac{\mathrm{r}_{\mathrm{i}}}{\mathrm{R}}\right)
$$

De acordo com a Eq. 5, o valor de $\mathrm{H}$ é independente da ordem seqüencial de $r_{i}$ na série temporal; assume o valor zero, quando $\mathrm{R}$ ocorre apenas uma vez no ano, e o valor máximo $\left(\log _{2} n\right)$, quando $R$ ocorre em todos os dias do ano. A entropia se aproxima do seu valor máximo quanto mais uniforme for sua distribuição, ou seja, quando os dados da série apresentam pouca variabilidade temporal. Nesse contexto, pode-se interpretar H como uma estimativa da variabilidade da precipitação pluvial no sentido de escala.

Quando as séries de precipitação anuais para $\mathrm{n}$ anos estão disponíveis, para um mesmo posto pluviométrico, a melhor estimativa da entropia anual pode ser obtida através da média aritmética das entropias do período analisado, como:

$$
\overline{\mathrm{H}}=\frac{1}{\mathrm{n}} \sum_{\mathrm{i}=1}^{\mathrm{n}} \mathrm{H}
$$


em que:

$\overline{\mathrm{H}}$ - entropia média, bits

n - número de anos que foram utilizados no cálculo de $\mathrm{H}$, adimensional

O ganho de uma informação resulta no decréscimo da entropia e vice-versa. A entropia se torna nula quando existe certeza absoluta da ocorrência de um evento ou, estatisticamente, quando todas as probabilidades de um conjunto, exceto uma, é zero.

No presente trabalho foram elaborados mapas de isoentropias correspondentes ao período anual e aos trimestres mais secos e mais chuvosos para o Estado da Paraíba, com vistas à quantificação da variabilidade espacial da precipitação pluvial.

\section{RESULTADOS E DISCUSSÃO}

Os mapas de isoentropias anual e dos trimestres mais secos e mais chuvoso do Estado da Paraíba são apresentados na Figura 1. A análise das isolinhas correspondentes à média anual de entropia revela máximos praticamente em todo o Litoral do Estado e na microrregião da Borborema, com valores superiores a 6,0 bits, e mínimos no Cariri e Curimataú, com isolinhas de até 3,9 bits (Figura 1A). As microrregiões com entropia mínima são também as que apresentaram menores totais de precipitação no Estado, como é o caso de Cabaceiras, localizada no Cariri Paraibano, conhecida como a cidade que menos chove em todo o Brasil, com média climatológica de pouco mais de $300 \mathrm{~mm}$ anuais. Nas microrregiões do Litoral, Brejo, Agreste e pequena parte do Sertão (Oeste do Estado) a entropia foi superior a 5,0 bits. Em grande parte desta última microrregião obtiveram-se isoentropias com valores intermediários entre 4,0 e 5,0 bits, significando que a variabilidade da precipitação pluvial anual do Estado da Paraíba aumenta do Litoral para o Sertão, sendo mínima na Zona da Mata e máxima no Cariri e Curimataú. Este padrão de comportamento da isoentropia também é observado no trimestre seco (Figura 1B). A entropia decresceu da Zona da Mata para o Sertão, com máximos no Litoral Norte, pequena parte do Litoral Sul e na microrregião da Borborema, com valores de aproximadamente 0,4 bits. Ainda no trimestre seco, o menor valor da entropia foi de 0,2 bits, entre a microrregião da Borborema e o Oeste do Estado. Esta análise evidencia que, durante a estação seca, a variabilidade da precipitação pluvial é menor no Litoral e maior no Oeste do Estado. Os baixos valores apresentados da entropia para esse período são atribuídos à alta variabilidade da precipitação pluvial. No trimestre chuvoso (Figura 1C), onde ocorre menor variabilidade na precipitação pluvial, quando comparados com os do trimestre seco (Figura 1B), observam-se valores mais elevados de isoentropias na parte Oeste que no Litoral do Estado. Kawachi et al. (2001) obtiveram valores de entropia que variaram de 5,7 a 6,7 bits quando analisaram o padrão de comportamento da precipitação pluvial no Japão.

O mapa de isoentropias do trimestre chuvoso, exibido na Figura 1C, apresenta máximos de entropia no Oeste e mínimos no Litoral do Estado. Esse comportamento é aparentemente conflitante quando comparado com a distribuição de entropia anual e do trimestre seco; entretanto, para ilustrar as distribui-
A.

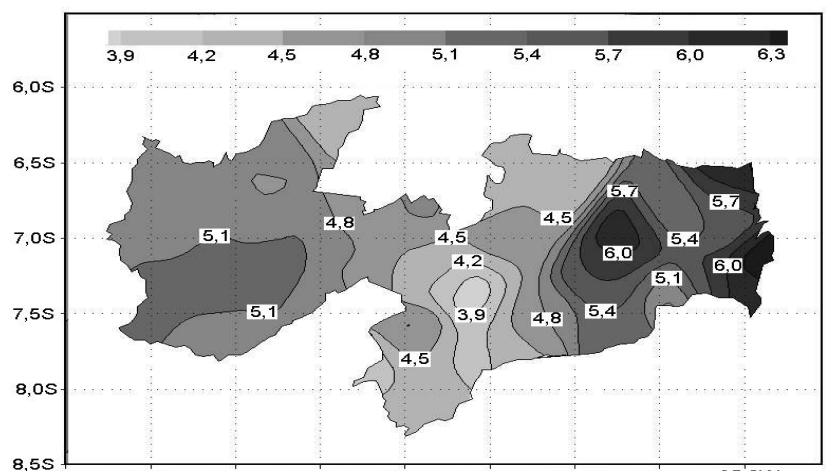

B.

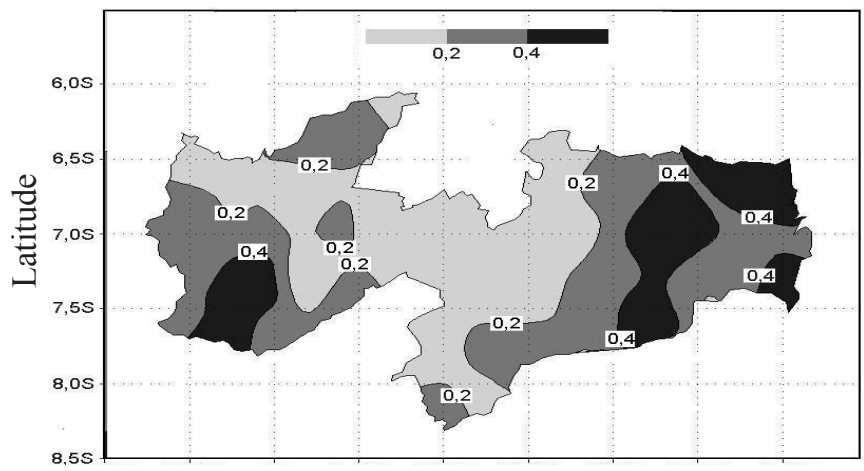

C.

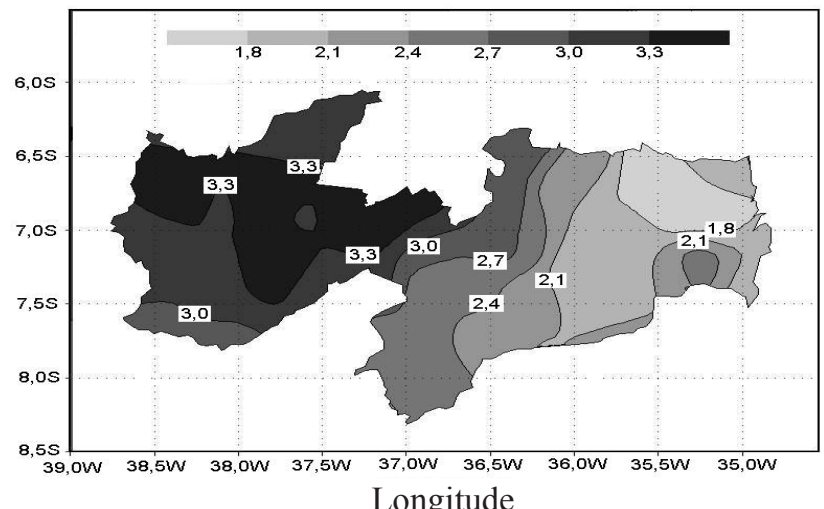

Figura 1. Mapa de isoentropias anual (A), trimestre seco (B) e trimestre chuvoso (C) do Estado da Paraíba, com base na média de dez anos de dados

ções de precipitação pluvial e de entropia nessas regiões, a Tabela 2 exibe a percentagem dos totais pluviométricos dos trimestres secos e chuvosos e as médias da entropia anual e dos dois trimestres, correspondentes a seis localidades do

Tabela 2. Precipitação pluvial dos trimestres (Prec-T) chuvosos e secos, em relação ao total, em percentagem, entropia dos trimestres (Ent-T) chuvosos e secos e entropia anual (EntAnual), em bits, em seis localidades do Estado da Paraíba

\begin{tabular}{llllllll}
\hline \multirow{2}{*}{ Localidade } & Período Período & \multicolumn{2}{c}{ Prec-T (\%) } & & \multicolumn{3}{c}{ Ent-T (bit) } \\
\cline { 3 - 7 } \cline { 5 - 7 } & Chuvoso Seco & \multicolumn{2}{c}{ Chuvoso Seco } & Chuvoso Seco Anual \\
\hline Araçagi & Mai-Jul Out-Dez & 47,16 & 6,59 & 2,32 & 0,39 & 5,10 \\
B. B. Cruz & Fev-Abr Ago-Out & 74,34 & 1,46 & 3,03 & 0,05 & 4,20 \\
Aguiar & Fev-Abr Ago-Out & 67,78 & 2,63 & 3,33 & 0,19 & 5,14 \\
Areia & Mai-Jul Out-Dez & 44,84 & 6,12 & 2,74 & 0,44 & 6,20 \\
Aparecida & Fev-Abr Ago-Out & 67,36 & 1,33 & 3,28 & 0,08 & 5,05 \\
Alhandra & Mai-Jul Set-Nov & 46,10 & 5,44 & 2,68 & 0,43 & 6,03 \\
\hline
\end{tabular}


Estado da Paraíba, sendo três delas localizadas no Litoral e no Brejo Paraibano (Araçagi, Areia e Alhandra) e as outras no Sertão (Belém do Brejo do Cruz, Aguiar e Aparecida).

Nas localidades de Araçagi, Areia e Alhandra, pertencentes às microrregiões do Litoral e Brejo Paraibano, a precipitação pluvial do trimestre chuvoso foi inferior a $50 \%$ do total anual e a entropia foi, respectivamente, 2,32; 2,74 e 5,44 bits. Nas localidades de Belém do Brejo do Cruz, Aguiar e Aparecida, localizadas no Sertão Paraibano, a precipitação nesse trimestre foi em torno de $70 \%$ do total anual e a entropia foi, respectivamente, 3,03; 3,33 e 3,28 bits. Nas microrregiões do Oeste do Estado, a maior parte da precipitação pluvial anual se concentra no trimestre chuvoso, enquanto no Brejo e Litoral a chuva é melhor distribuída ao longo do ano. O percentual do total precipitado no trimestre seco foi maior nas localidades do Brejo e Litoral que nas localidades do Sertão. A entropia também se manteve maior nas localidades de maior pluviosidade nesse período. Ressalta-se que a precipitação média anual do Brejo e Litoral do Estado da Paraíba é superior a $1.500 \mathrm{~mm}$ e no Sertão, que se encontra inserido no interior do semi-árido nordestino, é inferior a $800 \mathrm{~mm}$ anuais. Em resumo, ao contrário da distribuição anual e do trimestre seco, a entropia da precipitação no trimestre chuvoso nas localidades do Sertão é maior que a entropia das localidades do Litoral e Brejo Paraibano, embora a precipitação média anual dessas microrregiões seja bastante superior à do Sertão. Assim, no trimestre chuvoso a variabilidade da precipitação é maior no Litoral e Brejo e menor no Sertão, justamente o inverso do comportamento anual e do trimestre seco. Esses resultados indicam que a entropia da precipitação é maior nos locais e períodos de maior pluviosidade e menor quando esses locais e períodos estão sujeitos a baixos índices de precipitação pluvial. A evidência desse resultado leva à constatação de que a variabilidade da precipitação pluvial no Estado da Paraíba é menor no Litoral e Brejo e maior no Sertão, durante o ano e o trimestre seco. Por outro lado, durante o trimestre chuvoso a variabilidade é maior no Litoral e Brejo e menor no Sertão.

O comportamento temporal anual e intra-anual da entropia da precipitação pluvial de Campina Grande, PB, é apresentado na Figura 2. No período analisado, a média da entropia foi 5,85 bits; os máximos, com valores superiores a 6,0 bits, ocorreram nos anos chuvosos de 1940, 1959, 1963 e 1964 e mínimos nos anos secos de 1953 e 1970, com valores de 5,38 e 5,27 bits, respectivamente (Figura 2A). A entropia intra-anual da precipitação pluvial apresenta alta variabilidade, com valores máximos nos meses chuvosos e mínimos nos meses secos (Figura 2B). Essa configuração se assemelha à distribuição de freqüência da precipitação pluvial de Campina Grande, com os meses chuvosos e secos centrados exatamente nos períodos de máxima e de mínima entropia, respectivamente.

A entropia da temperatura média do ar em Campina Grande apresenta-se constante, com valor máximo em torno de 8,53 bits, ao longo de todo o período estudado (Figura 3A). Isso ocorreu pelo fato de que a variação da temperatura do ar é muito pequena, ao contrário de outras variáveis climatológicas, como a precipitação pluvial, e a teoria da entropia expressa o grau de contrastes da série temporal dos dados. Pelo mesmo

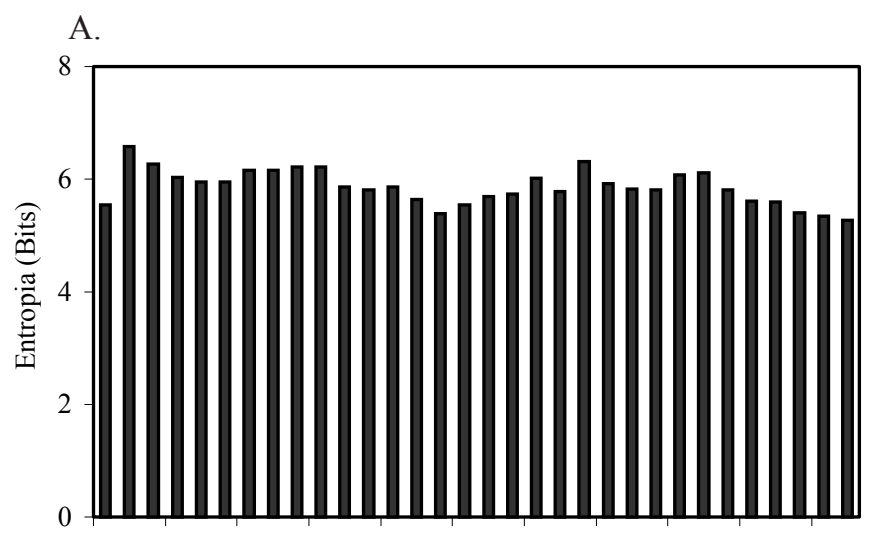

19391942194519481951195419571960196319661969

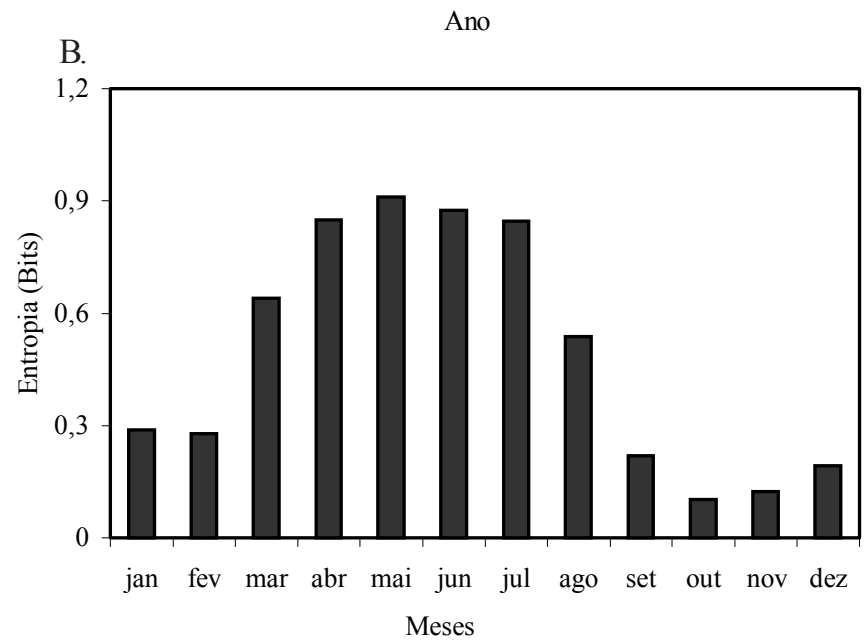

Figura 2. Comportamento temporal anual (A) e intra-anual (B) da entropia da precipitação pluvial em Campina Grande, PB, no período de 1939 a 1972

motivo, a entropia intra-anual da temperatura apresenta baixa variabilidade, com mínimo nos meses frios, de julho a agosto, e máximo nos meses mais quentes do ano (Figura 3B).

A relação entre a entropia da série temporal de precipitação pluvial de Campina Grande, com o seu desvio-padrão, é apresentada na Figura 4. À medida em que aumenta o grau de contraste dos dados da série temporal, o desvio-padrão também aumenta e a entropia da variável estudada diminui exponencialmente. A entropia é máxima ( 8,53 bits) quando todos os dados da série temporal são rigorosamente iguais e diminui quando a semelhança entre os dados é reduzida. Por outro lado, a relação entre a entropia da temperatura do ar e o desvio-padrão da série temporal apresenta exatamente o mesmo comportamento. Esse resultado comprova aqueles obtidos por Montaño et al. (2001), em que o valor da entropia decresce com o aumento do número de contraste e aumenta com o decréscimo desse número. Assim, a entropia pode ser considerada uma estimativa funcional da incerteza associada à distribuição de probabilidade.

Esses resultados evidenciam que nos meses chuvosos a entropia da precipitação pluvial é alta porque os desvios-padrão das séries temporais correspondentes são menores quando comparados com aqueles dos anos e meses secos, que têm, ao contrário, baixa entropia e alto desvio-padrão. A variação intraanual da temperatura apresenta comportamento semelhante, 

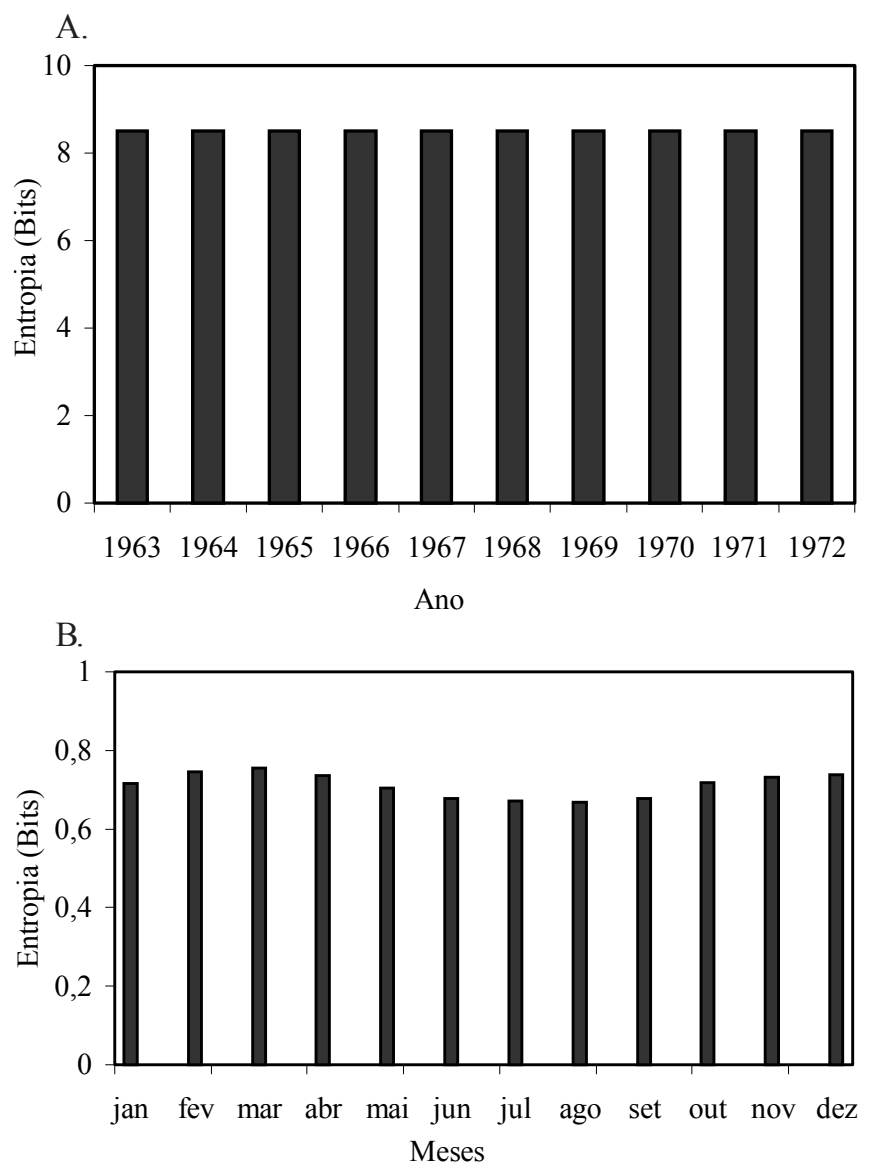

Figura 3. Comportamento temporal anual (A) e intra-anual (B) da entropia da temperatura média do ar em Campina Grande, no período de 1963 a 1972

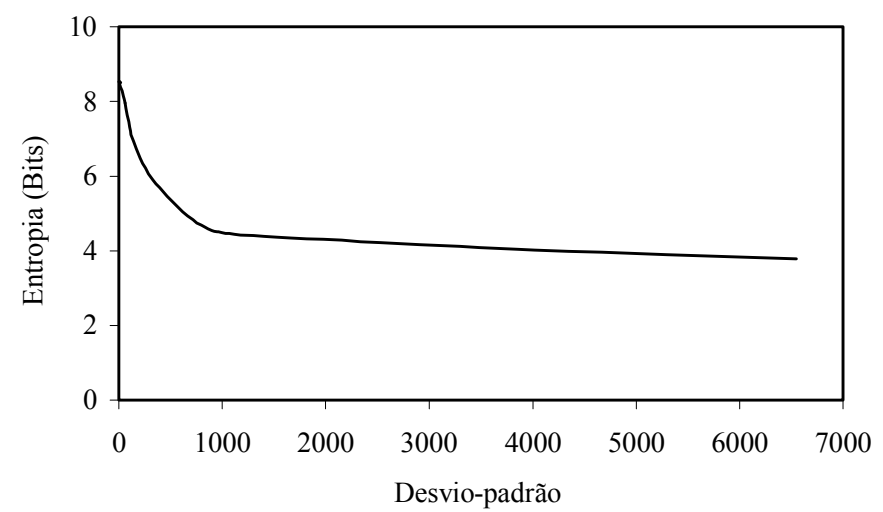

Figura 4. Relação entre a entropia da informação e o desviopadrão de uma série temporal (precipitação pluvial ou temperatura do ar)

ou seja, os meses com baixa temperatura têm desvio-padrão maior porque a entropia é menor que nos meses de verão. Assim, a variabilidade da precipitação pluvial é menor e maior, respectivamente, nos anos e meses chuvosos e secos.

\section{CONCLUSÕES}

1. A entropia da precipitação pluvial no Estado da Paraíba é maior nos locais e períodos de maior pluviosidade (Litoral e Brejo, no período de maio a julho) e menor nas áreas onde chove menos e nos períodos de estiagem (Cariri e Sertão, no período de agosto a outubro).

2. A variabilidade da precipitação pluvial na Paraíba é menor no Litoral e Brejo e maior no Sertão, durante o ano e trimestre seco, enquanto durante o trimestre chuvoso a variabilidade é maior no Litoral e Brejo e menor no Sertão.

3. A baixa variabilidade da temperatura média do ar é responsável pelo comportamento anual constante da entropia, com o valor máximo de 8,53 bits, ao longo do período estudado.

4. A entropia decresce exponencialmente com o aumento do desvio-padrão de qualquer série temporal de dados.

\section{LITERATURA CITADA}

Cavalcanti, E.P.; Silva, E.D.V. Estimativa da temperatura do ar em função das coordenadas locais. In: Congresso Brasileiro de Meteorologia, 8, e Congresso Latino-Americano de Ibérico de Meteorologia, 2, 1994, Belo Horizonte. Anais... Belo Horizonte: SBM, 1994. v.1, p.154-157.

Chapman, T.G. Entropy as a measure of hydrologic data uncertainty and model performance. Journal of Hydrology, Amsterdam, v.85, n.1, p.111-126, 1986.

Dragomir, S.S.; Scholz, M.L.; Sunde, J. Some upper bounds for relative entropy and applications. Journal Computers and Mathematics with Applications, Oxford, v.39, n.1, p.91-100, 2000.

Kaberger, T.; Mansson, B. Entropy and economic processes physics perspectives. Ecological Economics, Amsterdam, v.36, n.1, p.165-179, 2001

Kawachi, T.; Maruyama, T.; Singh, V.P. Rainfall entropy delineation of water resources zones in Japan. Journal of Hydrology, Amsterdam, v.246, n.1, p.36-44, 2001.

Montaño, M.A.J.; Ebeling, W.; Pohl, T.; Rapp, P.E. Entropy and complexity of finite sequences as fluctuating quantities. BioSystems, Oxford, v.64, n.1, p.23-32, 2001.

Rajagopal, A.K.; Teitler, S.; Singh, V.P. Some new perspectives on maximum entropy techniques in water resources research. In: Singh, V. P. (Ed) Hydrologic frequency modeling. Dordrechet: D. Reijel Publishing, 1987, p.247-366

Ricotta, C. Bridging the gap between ecological diversity indices and measures of biodiversity with Shannon's entropy: comment to Izák and Papp. Ecological Modeling, Amsterdam, v.46, n.1, p.1-3, 2001.

Roucou, P.; Aragão, J.O.R.; Harzallah, A.; Fontaine, B.; Janicot, S. Vertical motion, changes to Northeast Brazil rainfall variability: A GCM simulation. International Journal of Climatology, West Sussex, v.16, n.1, p.879-891, 1996.

Shannon, C.E.; Weaver, W. The mathematical theory of communications. Urbana: University Illinois Press, 1949, 125p.

Silva, V.P.R.; Azevedo, P.V.; Brito, J.I.B. Origem do máximo relativo de pluviometria no extremo Oeste do Estado da Paraíba. In: Congreso Argentino de Meteorología, 7, Congreso, e Latinoamericano e Ibérico de Meteorología, 7, 1996, Buenos Aires, Anais... Buenos Aires: Centro Argentino de Meteorológos y Federación Latinoamericana Y Ibérica de Sociedade de Meteorologia, 1996. p.101-102.

Singh, V.P. The use of entropy in hydrology and water resources. Hydrological Processes, Bristol, v.11, n.1, p.587-626, 1997.

Sonuga, J.O. Entropy principle applied to the rainfall-runoff process. Journal of Hydrology, Amsterdam, v.30, n.1, p.8194, 1976. 